\section{miR-101 suppresses the development of MLL-rearranged acute myeloid leukemia}

Dysregulation of miRNA (a class of short non-coding RNA) has been observed in solid tumors and leukemia, ${ }^{1,2}$ and the role of miRNA in cancer development is largely context-dependent. ${ }^{2}$ While several miRNA (e.g. miR$29 \mathrm{~b})^{3}$ have been functionally linked to acute myeloid leukemia (AML), their antitumor effects in vivo are not evident, possibly due to the complexity and diversity of miRNA-mediated gene regulation. It remains unclear how miRNA contribute to an aggressive phenotype in heterogenous AML. Using an integrated miRNA and mRNA expression analysis, here we uncover a miRNAregulatory network composed of eight miRNA (i.e. miR29a/b, miR-101, miR-222, miR-26b, miR-27b, miR-140 and miR-155) whose downregulation is associated with leukemia aggressiveness. We also define a tumor suppressive role for miR-101 in the development of mixedlineage leukemia (MLL)-rearranged AML. Restoration of miR-101 expression significantly impedes leukemia initiation and progression through induction of cell cycle inhibitors and inhibition of genes associated with selfrenewal and pro-survival in leukemic stem cells (LSC).
Oncogenic rearrangement of the MLL gene in hematopoietic stem/progenitor cells (HSPC) induces aberrant gene expression and confers a poor clinical prognosis in acute leukemias. Transformation by MLL fusion proteins is primarily mediated through direct upregulation of the HOXA-cluster genes and HOX co-factors such as MEIS1. ${ }^{4}$ HOXA9 and MEIS1 are the most essential downstream effectors of MLL fusion proteins, and we have previously reported that their co-expression is sufficient to predispose HSPC to malignant transformation and induce AML. ${ }^{5}$ Aberrant overexpression of HOXA9 and MEIS1 is required for the induction and maintenance of $M L L$-rearranged AML, where LSC have been functionally defined. ${ }^{4,5}$

We and others have shown that MLL fusion proteins (e.g., MLL-AF9) can serve as an initiating event for oncogenic conversion of normal HSPC into pre-LSC, which subsequently acquire additional events upon transplantation into mice for the development of LSC. ${ }^{4-7}$ The selfrenewal rate in a pre-leukemic clone determines subsequent tumorigenic potential in vivo. We have previously demonstrated that pre-LSC transformed from normal HSPC by MLL-AF9 have higher self-renewal potential and produce a more aggressive leukemia in mice with shorter latency than pre-LSC transformed by
A

Transduction

MLL-AF9 versus Hoxa9/Meis1
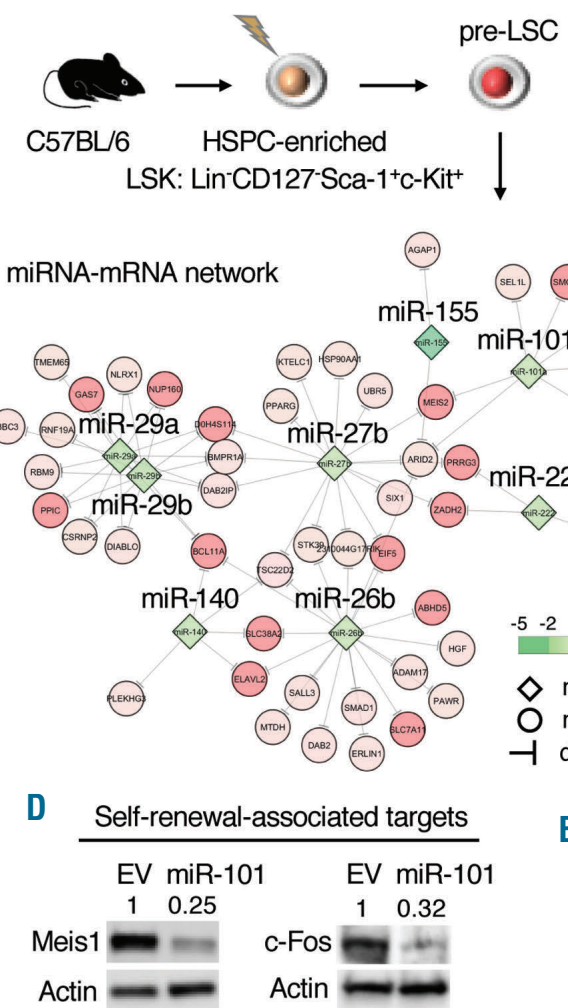

$\mathrm{F}$

Cell cycle analysis $(n=3)$

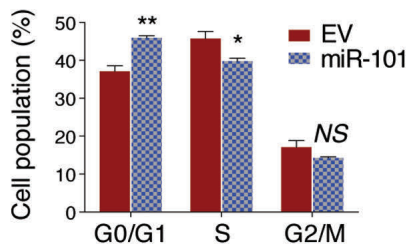

B Serial colony replating - Week 6
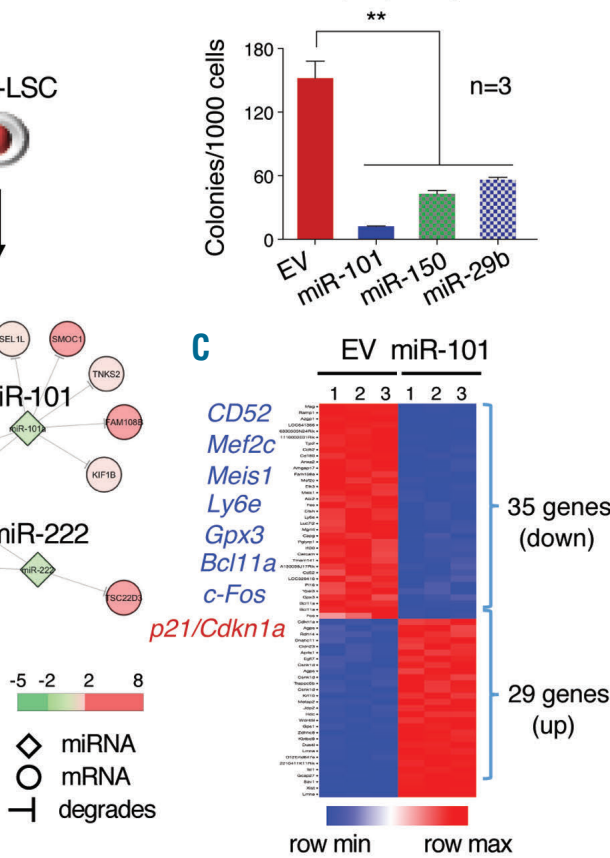

E Apoptosis $(n=3)$

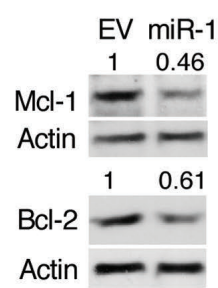

Cell-cycle inhibitors

\begin{tabular}{rr}
\hline EV miR-101 & EV miR-101 \\
p21 & p27 $=-1.94$ \\
Actin & Actin $=\infty$
\end{tabular}

Figure 1. Overexpression of miR-101 impairs the function of MLL-AF9 preleukemic stem cells (pre-LSC). (A) Integrated genomic analysis identifying a tumor suppressor miRNA-regulatory network in MLL-AF9 pre-LSC compared to HOXA9/MEIS1 pre-LSC. Diamond shape: miRNA identified by Exiqon miRCURY LNA microRNA array. Circle: mRNA (targeted by miRNA) identified by Illumina expression array. (B) Serial colony replating assay of miRNA-expressing MLL-AF9 preLSC. The number of colonies per dish at the $6^{\text {th }}$ round of replating is shown $(n=3)$. (C) Heat map of microarray analysis showing differential gene expression in MLL-AF9 pre-LSC overexpressing miR-101 versus empty vector (EV) control $(n=3)$ with a cut-off of the false discovery rate (FDR) $\leq 0.05$ and fold change $(F C) \geq 1.5$. (D) Western blot analysis confirming miR101-induced downregulation of Meis1 and c-Fos expression in MLLAF9 pre-LSC. (E) Percentage of apoptotic cells $(n=3)$ in MLL-AF9 pre-LSC and western blot analysis showing reduced expression of $\mathrm{Mcl}-1$ and $\mathrm{Bcl}-$ 2 by miR-101 overexpression. (F) Cell cycle analysis of miR-101-expressing MLL-AF9 pre-LSC using $\mathrm{Ki}-67$ and 7AAD staining $(n=3$ independent experiments) and western blot analysis revealing miR-10-induced upregulation of p21 and p27 expression. $* P<0.05 ; * * P<0.01$; NS: not significant $(P>0.05)$. 
HOXA9/MEIS1. ${ }^{5,7}$ Despite major differences in selfrenewal and mouse survival, pre-LSC mediated by MLLAF9 and HOXA9/MEIS1 display similar immunophenotype and induce AML with similar histopathologic manifestations. ${ }^{5,7}$ Thus, genes differentially expressed in MLLAF9 versus HOXA9/MEIS1 pre-LSC may contribute to the highly aggressive phenotype in MLL-AF9-induced AML.

To identify miRNAs that regulate the differential gene expression, we performed an integrated analysis for miRNA and mRNA expression profiling using the Bayesian Network with Splitting-Averaging strategy, and identified a tumor suppressive miRNA-regulatory network in HSPC-derived MLL-AF9 versus HOXA9/MEIS1 pre-LSC ${ }^{4,7}$ (Figure 1A and Online Supplementary Figure $S 1 A-D)$. Of the eight miRNA identified, miR-101, miR-29b, miR-222 and miR-155 are reportedly down-regulated in newly-diagnosed AML patient samples compared to normal human $\mathrm{CD}_{3} 4^{+}$ HSPC, while miR-29a and miR-29b are down-regulated in patients with MLL-rearranged AML compared to other AML subtypes displaying a subtype-specific feature. These observations are consistent with our result showing lower levels of these miRNA in LSC (L-GMP, $\left.\mathrm{Lin}^{-} \mathrm{CD} 127^{-}{ }^{-} \mathrm{c}-\mathrm{Kit}^{+} \mathrm{Sca} 1^{-} \mathrm{GFP}^{+} \mathrm{CD} 16 / 32^{\text {high }} \mathrm{CD} 34^{+}\right)^{4}$ flowsorted from mice with MLL-AF9-induced AML than in normal murine HSPC (Online Supplementary Figure S1E), supporting a potential tumor suppressor role for the miRNA-regulatory network in MLL-rearranged AML.

Among the eight miRNA, miR-29b has been functionally defined as a tumor suppressor and its overexpression reduced tumorigenicity in a BCR-ABL-expressed $\mathrm{K} 562$ xenograft mouse model of human chronic myeloid

A Survival - Primary AML B Primary AML-BM C Primary AML LSC $(n=6)$
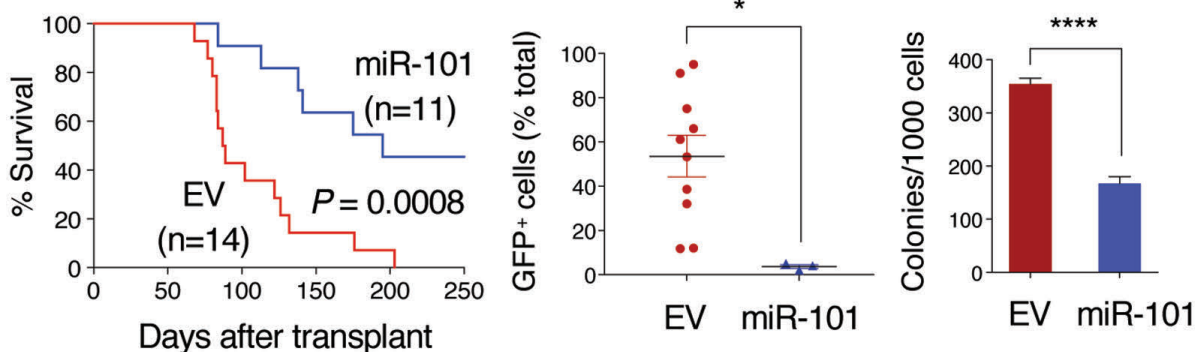

D

Primary AML LSC

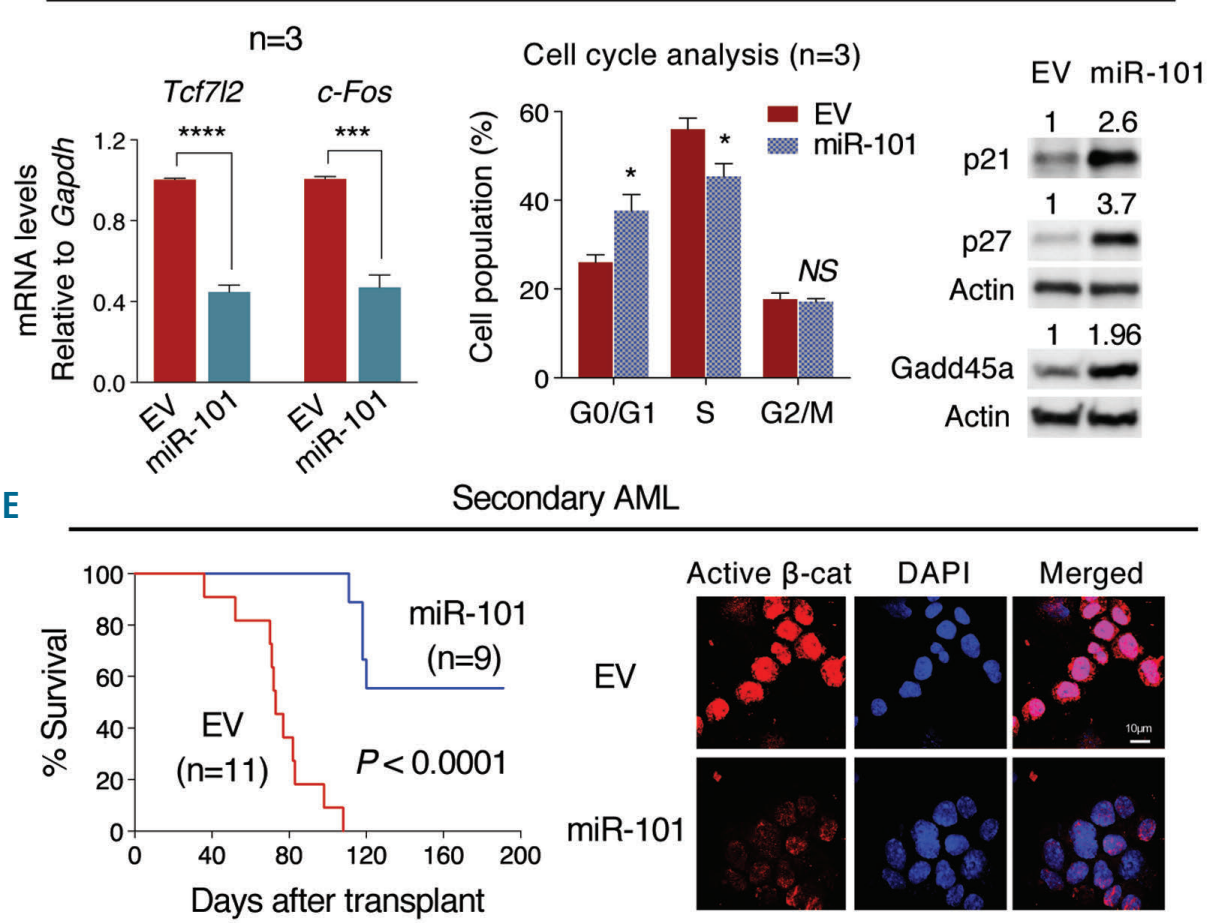

Figure 2. miR-101 suppresses the development of leukemic stem cells (LSC) in a mouse model of MLL-AF9-driven acute myeloid leukemia (AML). (A) KaplanMeier survival curves of mice receiving miR-101-expressing MLL-AF9 pre-LSC. 1x10 6 pre-LSC were transplanted into sublethally irradiated (6 Gy) BL6 recipient mice for the development of primary AML. P-values were determined by the log-rank test. (B) Percentages of GFP-positive $\left(^{+}\right)$leukemic cells in the bone marrow (BM) of mice with primary AML. (C) Colony forming assay of MLL-AF9 LSC from primary AML. (D) Real-time quantitative polymerase chain reaction analysis of Tcf7/2 and c-Fos gene expression, cell cycle analysis and expression of p21, p27, and Gadd45a proteins in primary MLL-AF9 LSC. (E) Kaplan-Meier survival curves of mice receiving GFP+ ${ }^{+}$MLL-AF9 leukemic cells isolated from primary AML and confocal immunofluorescence showing miR-101-induced reduction of nuclear active $\beta$-catenin in leukemic cells from secondary AML. $* P<0.05 ; * \star * P<0.0005 ; * * * * P<0.0001$; NS: not significant $(P>0.05)$. 

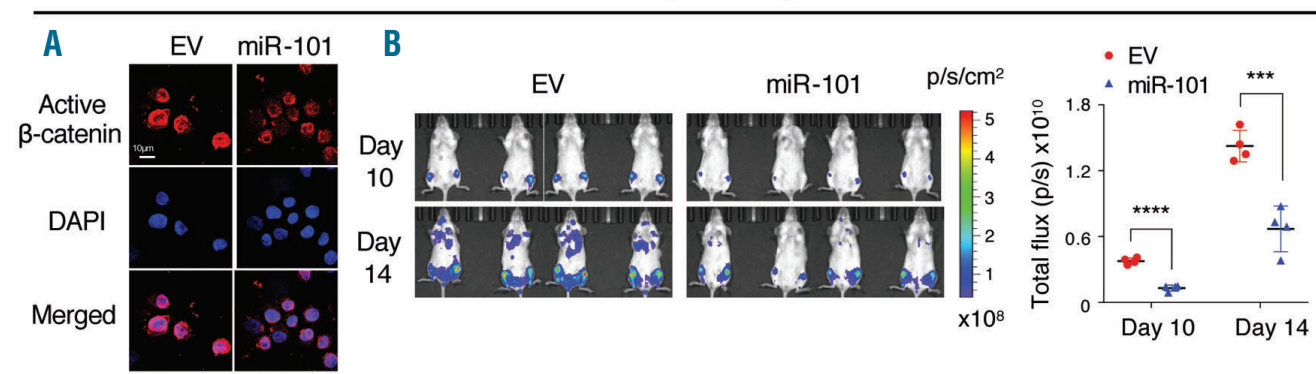

MOLM-13 xenograft - Day14 post-transplantation
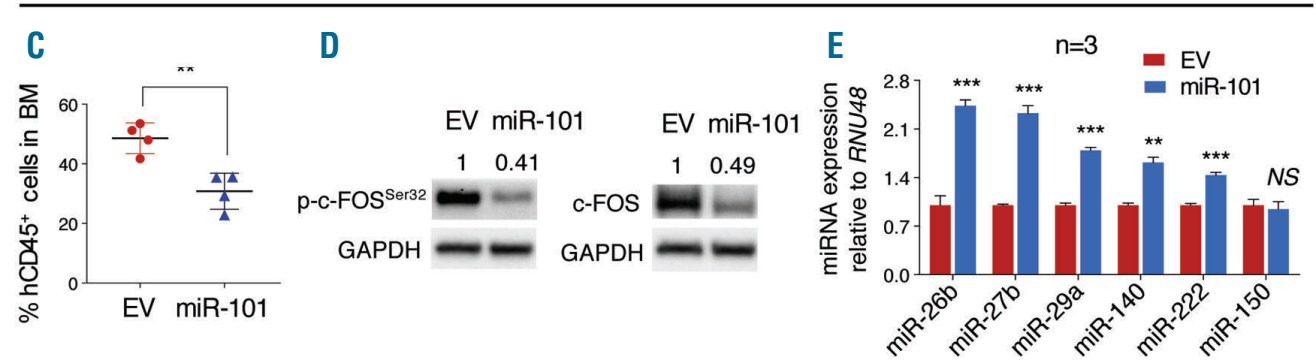

Figure 3. miR-101 reduces tumor burden in human MLL-AF9 (MOLM-13) xenografts. (A) Confocal immunofluorescence confirming miR-101-induced decrease of nuclear active $\beta$-catenin in human MLL-AF9 (MOLM-13) acute myeloid leukemia (AML) cells. (B) Bioluminescence imaging and total flux (photons/sec; $\mathrm{p} / \mathrm{s}$ ) of MOLM-13 xenograft mice $(n=4)$. (C) Percentage of $h C D 45^{+}$cells engrafted in the bone marrow (BM) of MOLM-13 xenograft mice. (D) Western blot analysis showing miR-101-induced reduction in p-c-FOS ${ }^{\text {Ser32 }}$ and c-FOS levels. (E) Real time-quantitative polymerase chain reaction analysis of miRNA expression in miR-101expressing human MLL-AF9 AML cells isolated from the BM of MOLM-13 xenograft mice. $* * P<0.01 ; * * * P<0.0005$; $* * * * P<0.0001$; NS: not significant $(P>0.05)$.

leukemia (CML) by targeting apoptosis, cell cycle and proliferation pathways. ${ }^{3}$ While miR-101 is reported as a putative tumor suppressor in several types of cancer via targeting diverse oncogenic pathways, ${ }^{10}$ its role in AML has not yet been explored. To further evaluate the miRNA-regulatory network, we investigated the tumor suppressive function of miR-101, which was one of the most down-regulated miRNA identified, in MLL-AF9induced AML. We used miR-150 as a control because, despite not being in the network, its expression is downregulated in both AML and CML. ${ }^{11}$

HSPC-derived MLL-AF9 pre-LSC were transduced with a retroviral vector expressing miR-101, miR-29b or miR-150. Ectopic expression of these miRNA reduced the ability of pre-LSC to form colonies in serial replating assays (Figure $1 \mathrm{~B}$ and Online Supplementary Figure S2A and $B)$. Notably, miR-101 induced a significantly stronger growth-inhibitory effect on MLL-AF9 pre-LSC than miR$29 \mathrm{~b}$ and miR-150, which was correlated with miR-101mediated upregulation of cell-cycle inhibitor p21/Cdkn1a and downregulation of key Wnt/self-renewal target genes, including Meis1, c-Fos, Mef2c, Bcl11a, CD52, Gpx3 and Lybe identified by microarray analysis (Figure 1C). Subsequent RT-PCR and western blot analyses confirmed increased expression of $\mathrm{p} 21$ and reduced levels of Meis1, Bcl11a, c-Fos and Tcf712, which are known self-renewal genes in MLL-AF9-induced $\mathrm{AML}^{4,5,7}$ (Figure $1 \mathrm{D}$ and Online Supplementary Figure S2C). We and others have previously demonstrated that $\mathrm{Wnt} / \beta$-catenin signaling is required for the development of LSC in AML. ${ }^{5}$ Tcf712 and c-Fos are two key $\beta$-catenin transcriptional cofactors driving transcription of $\mathrm{Wnt} / \beta$-catenin target genes likely contributing to LSC self-renewal. ${ }^{7}$ Furthermore, the phenotypic defect in pre-LSC was accompanied by decreased cell proliferation in methylcellulose and induced apoptotic cell death through suppression of pro-survival proteins Mcl-1 and Bcl-2, as well as G1 cell cycle arrest through elevation of cell-cycle inhibitors p21 and p27 (Figure 1E and $\mathrm{F}$ and Online Supplementary Figure S2D). These findings suggest that restoring expression of miR-101 impairs pre-LSC functions through regulation of genes associated with Wnt/self-renewal, pro-survival and cell cycle pathways.

We next assessed the inhibitory effect of miR-101 overexpression on AML development by intravenously transplanting miR-101-expressing MLL-AF9 pre-LSC into sublethally irradiated syngeneic recipient mice. Our data showed that enforced expression of miR-101 reduced the incidence and delayed the onset and progression of AML in mice, which was accompanied by a significant decrease in leukemic cell infiltration in bone marrow (BM) (Figure 2A and B). miR-101-expressing MLL-AF9 LSC flow-sorted from primary AML revealed reduced colony-forming capacity (Figure 2C and Online Supplementary Figure S3). This was likely caused by miR101-induced suppression of Wnt target genes (Tcf 712 and $c$-Fos), and cell cycle arrest at the G1 phase via upregulation of p21, p27 and Gadd45a (Figure 2D). As a consequence of compromised LSC, miR-101-expressing AML cells from primary recipients generated a less aggressive leukemia in secondary recipient mice, whose BM cells exhibited a marked decrease in nuclear active $\beta$-catenin (Figure 2E). Altogether, these data underscore a tumor suppressor role for miR-101, whose ectopic expression impairs LSC development and reduces AML aggressiveness in mice.

We then investigated the effect of miR-101 overexpression on tumor burden in a mouse xenograft model of 
human MLL-AF9 (MOLM-13) AML, which has an extremely short latency (14 days) and is an effective xenotransplantation model for in vivo functional studies. Consistent with our observation in murine MLL-AF9 leukemic cells, stable overexpression of miR-101 substantially decreased nuclear active $\beta$-catenin in MOLM13 cells (Figure 3A and Online Supplementary Figure S4A). In vivo bioluminescence imaging showed that miR-101 overexpression reduced engraftment of human MOLM13 leukemic cells in NOD/SCID/IL2R gamma-null (NSG) mice without affecting bone marrow homing (Figure $3 \mathrm{~B}$ and $\mathrm{C}$ and Online Supplementary Figure S4B).

In agreement with miR-101-induced inhibition of leukemia cell proliferation in vivo, miR-101 markedly decreased the expression and phosphorylation of c-FOS (Figure 3D), a known $\beta$-catenin transcriptional co-factor implicated in the regulation of cell growth, survival, apoptosis, transformation and oncogenesis. ${ }^{12}$ Phosphorylation of c-FOS is essential for its protein stabilization and maximal transactivation contributing to its increased cell-transforming activity. ${ }^{13}$ c-FOS is reportedly a direct target of several miRNA, including miR-101, miR-29 and miR-222. ${ }^{14}$ Our data confirmed a miR-101mediated reduction in luciferase activity from the construct containing the c-FOS 3'-UTR (Online Supplementary Figure $S 4 C$ ). Enforced expression of exogenous c-FOS prevented miR-101-induced inhibition of leukemic cell viability (Online Supplementary Figure S4D and E). This result supports the role for c-FOS as a functional downstream target of miR-101.

Notably, restoration of miR-101 up-regulated the miRNA co-expression network, including miR-26b, miR27b, miR-29a, miR-140 and miR-222, but did not alter the expression of miR-150 (Figure 3E). miR-101 is reportedly a direct regulator of histone methyltransferase EZH2 and likely regulates the miRNA network through modulation of EZH2-mediated H3K27me3. EZH2 augments MLLAF9-initiated leukemogenesis by enhancing a myeloid differentiation block in AML. ${ }^{15}$ Our data showed that overexpression of miR-101 reduced levels of EZH2 and H3K27me3 in murine MLL-AF9 pre-LSC and human MLL-AF9 (MOLM-13) AML cells (Online Supplementary Figure $S 5 A$ ). Selective inhibition of EZH2 by EZH2 inhibitor EPZ-6438 caused a marked increase in miR-26b, miR-27b, miR-29b and miR-140 without affecting miR150 (Online Supplementary Figure S5B), underlining epigenetic regulation of the miRNA network. Interestingly, we also observed EZH2 inhibitor-induced elevation of miR101 (Online Supplementary Figure S5B). It is likely that there is a mutual regulation between miR-101 and EZH2mediated H3K27me3. Increased miR-101 suppresses H3K 27 me3 by directly targeting EZH2; conversely, reduced $\mathrm{H} 3 \mathrm{~K} 27 \mathrm{me} 3$ restores expression of the miRNA network including miR-101. These findings collectively suggest that miR-101 exerts its gene regulatory function in leukemogenesis, at least in part, by activating and co-operating with the network components. This co-operation between the miRNA allows for the simultaneous targeting of multiple oncogenic pathways that maximizes the tumor suppressive effect of miR-101.

In conclusion, we report here a miRNA-regulatory network associated with disease aggressiveness and demonstrate a tumor suppressor role for miR-101 in MLLrearranged AML. Enforced expression of miR-101 impairs LSC self-renewal and restrains leukemia development in mice. Together with prior findings showing a tumor suppressor role for miR-29b in leukemia, ${ }^{3}$ our data support the value of the miRNA co-expression network as potential novel targets for miRNA-based therapies in AML.

Estrella Gonzales-Aloy, ${ }^{1}$ Patrick Connerty, ${ }^{1}$ Basit Salik, ${ }^{1}$ Bing Liu, ${ }^{2}$ Andrew J. Woo, ${ }^{3,4}$ Michelle Haber, Murray D. Norris, Jianlong Wang and Jenny Y. Wang

'Cancer and Stem Cell Biology Group, Children's Cancer Institute, University of New South Wales, Sydney, Australia; ${ }^{2}$ Kids Cancer Alliance, Translational Cancer Research Centre for Kids, Cancer Institute New South Wales, Sydney, Australia; ${ }^{3}$ Harry Perkins Institute of Medical Research, QEII Medical Centre, Nedlands, the University of Western Australia, Crawley, Australia; ${ }^{4}$ Centre for Medical

Research, The University of Western Australia, Crawley, Australia; ${ }^{5}$ Children's Cancer Institute, University of New South Wales, Sydney, Australia and 'Department of Cell, Developmental and Regenerative Biology, Black Family Stem Cell Institute, Icahn School of Medicine at Mount Sinai, New York, NY, USA

Acknowledgments: we thank the staff of the Mark Wainwright Analytical Centre for their technical assistance.

Funding: JYW was supported by NHMRC APP1128824 and Tour de Cure Senior Research Grant 17-UNSW-RS-03.

Correspondence: JENNY Y. WANG.

jwang@ccia.unsw.edu.au.

doi:10.3324/haematol.2018.209437

Information on authorship, contributions, and financial \& other disclosures was provided by the authors and is available with the online version of this article at www. haematologica.org.

\section{References}

1. Lu J, Getz G, Miska EA, et al. MicroRNA expression profiles classify human cancers. Nature. 2005;435(7043):834-838.

2. Calin GA, Croce CM. MicroRNA signatures in human cancers. Nat Rev Cancer. 2006;6(11):857-866.

3. Garzon R, Heaphy CE, Havelange V, et al. MicroRNA 29b functions in acute myeloid leukemia. Blood. 2009;114(26):5331-5341.

4. Krivtsov AV, Twomey D, Feng Z, et al. Transformation from committed progenitor to leukaemia stem cell initiated by MLL-AF9. Nature. 2006;442(7104):818-822.

5. Wang Y, Krivtsov AV, Sinha AU, et al. The Wnt/beta-catenin pathway is required for the development of leukemia stem cells in AML. Science. 2010;327(5973):1650-1653

6. Lynch JR, Yi H, Casolari DA, et al. Gaq signaling is required for the maintenance of MLL-AF9 induced AML. Leukemia. 2016;30(8):17451748.

7. Dietrich PA, Yang C, Leung HH, et al. GPR84 sustains aberrant betacatenin signaling in leukemic stem cells for maintenance of MLL leukemogenesis. Blood. 2014;124(22):3284-3294.

8. Liu B, Li J, Tsykin A, et al. Exploring complex miRNA-mRNA interactions with Bayesian networks by splitting-averaging strategy. BMC Bioinformatics. 2009;10:408.

9. Garzon R, Volinia S, Liu CG, et al. MicroRNA signatures associated with cytogenetics and prognosis in acute myeloid leukemia. Blood. 2008;111(6):3183-3189.

10. Varambally S, Cao Q, Mani R-S, et al. Genomic Loss of microRNA101 Leads to Overexpression of Histone Methyltransferase EZH2 in Cancer. Science. 2008;322(5908):1695-1699.

11. He Y, Jiang X, Chen J. The role of miR-150 in normal and malignant hematopoiesis. Oncogene. 2013;33(30):3887-3893.

12. Eferl R, Wagner EF. AP-1: a double-edged sword in tumorigenesis. Nat Rev Cancer. 2003;3(11):859-868.

13. Sasaki T, Kojima H, Kishimoto R, et al. Spatiotemporal regulation of c-Fos by ERK 5 and the E3 ubiquitin ligase UBR1, and its biological role. Mol Cell. 2006;24(1):63-75.

14. Liu J-J, Lin X-J, Yang X-J, et al. A novel AP-1/miR-101 regulatory feedback loop and its implication in the migration and invasion of hepatoma cells. Nucleic Acids Res. 2014;42(19):12041-12051.

15. Tanaka S, Miyagi S, Sashida G, et al. Ezh2 augments leukemogenicity by reinforcing differentiation blockage in acute myeloid leukemia. Blood. 2012;120(5):1107-1117. 